\title{
Correction to: Mechanical factors contributing to the Venus flytrap's rate-dependent response to stimuli
}

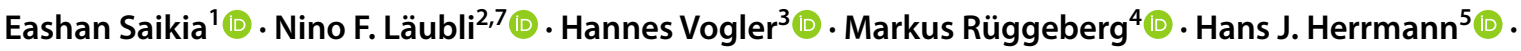

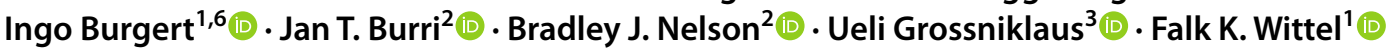

Published online: 21 October 2021

(c) The Author(s) 2021

\section{Correction to: Biomechanics and Modeling in Mechanobiology https://doi.org/10.1007/s10237-021-01507-8}

In the original publication of the article, the affiliation "Department of Mechanical and Process Engineering, ETH Zurich, Zurich 8092, Switzerland" was missed for the author "Nino F Läubli".

The author is affiliated with both "Department of Mechanical and Process Engineering, ETH Zurich, Zurich 8092, Switzerland" and "Department of Chemical Engineering and Biotechnology, University of Cambridge, Cambridge CB3 0AS, United Kingdom".

The author names in the citations were incorrectly placed outside the brackets. And in several instances the author names of the citation were repeated.

In the eighth reference, the author name was incorrectly given as "Forterre Yoë", and it should be "Forterre Yoël".

The original article has been corrected.
The original article can be found online at https://doi.org/10.1007/ s10237-021-01507-8.

\section{Eashan Saikia}

esaikia@ethz.ch

Falk K. Wittel

fwittel@ethz.ch

1 Department of Civil, Environmental and Geomatic Engineering, ETH Zurich, 8093 Zurich, Switzerland

2 Department of Mechanical and Process Engineering, ETH Zurich, 8092 Zurich, Switzerland
Open Access This article is licensed under a Creative Commons Attribution 4.0 International License, which permits use, sharing, adaptation, distribution and reproduction in any medium or format, as long as you give appropriate credit to the original author(s) and the source, provide a link to the Creative Commons licence, and indicate if changes were made. The images or other third party material in this article are included in the article's Creative Commons licence, unless indicated otherwise in a credit line to the material. If material is not included in the article's Creative Commons licence and your intended use is not permitted by statutory regulation or exceeds the permitted use, you will need to obtain permission directly from the copyright holder. To view a copy of this licence, visit http://creativecommons.org/licenses/by/4.0/.

Publisher's Note Springer Nature remains neutral with regard to jurisdictional claims in published maps and institutional affiliations.
3 Department of Plant and Microbial Biology and Zurich-Basel Plant Science Center, University of Zurich, 8008 Zurich, Switzerland

4 Institut Für Holztechnologie, 01217 Dresden, Germany

5 Laboratoire de Physique et Mécanique des Milieux Hétérogènes, École Supérieur de Physique et de Chimie Industrielles de la Ville de Paris, 75005 Paris, France

6 Swiss Federal Laboratories for Material Science and Technology-EMPA, Cellulose and Wood Materials Laboratory, 8600 Dubendorf, Switzerland

7 Department of Chemical Engineering and Biotechnology, University of Cambridge, Cambridge CB3 0AS, UK 